\section{Zebrafish-on the move towards ophthalmological research}

J Chhetri, G Jacobson and N Gueven

\begin{abstract}
Millions of people are affected by visual impairment and blindness globally, and the prevalence of vision loss is likely to increase as we are living longer. However, many ocular diseases remain poorly controlled due to lack of proper understanding of the pathogenesis and the corresponding lack of effective therapies. Consequently, there is a major need for animal models that closely mirror the human eye pathology and at the same time allow higher-throughput drug screening approaches. In this context, zebrafish as an animal model organism not only address these needs but can in many respects reflect the human situation better than the current rodent models. Over the past decade, zebrafish have become an established model to study a variety of human diseases and are more recently becoming a valuable tool for the study of human ophthalmological disorders. Many human ocular diseases such as cataract, glaucoma, diabetic retinopathy, and age-related macular degeneration have already been modelled in zebrafish. In addition, zebrafish have become an attractive model for pre-clinical drug toxicity testing and are now increasingly used by scientists worldwide for the discovery of novel treatment approaches. This review presents the advantages and uses of zebrafish for ophthalmological research.

Eye (2014) 28, 367-380; doi:10.1038/eye.2014.19; published online 7 February 2014
\end{abstract}

Keywords: zebrafish; vision; visual behaviour; ocular diseases; retina; drug development

\section{Overview}

Visual impairment is a major health problem affecting millions of people globally. ${ }^{1}$ Major causes of visual impairment and blindness include uncorrected refractive index, cataract, glaucoma, age-related macular degeneration
(AMD), and diabetic retinopathy (DR), with cataracts being the leading cause of blindness globally. ${ }^{1}$ The worldwide economic burden of vision loss is estimated to be about US \$3 trillion in 2010 and is anticipated to increase significantly by $2020 .^{2}$ Therefore, strategies to develop animal models that closely mirror human ocular diseases are necessary for a better understanding of the underlying causes and in parallel for the development of novel therapeutic approaches. Over the past decade, zebrafish have become an established model to study a variety of human diseases and are more recently becoming a valuable tool for the study of human ophthalmological disorders. ${ }^{3-6}$ Due to many advantages inherent to this model organism, zebrafish are also highly suitable for screening approaches to identify novel therapeutics in the field of ophthalmological medicine.

\section{Zebrafish as an animal model}

Over the past 20 years zebrafish (Danio rerio) have been increasingly used as a vertebrate model for developmental and genetic studies. ${ }^{7}$ There are many reasons for their popularity. Zebrafish are easy to maintain and breed in large numbers at relatively low cost. They become sexually mature after 3-4 months and each pair can generate 200-300 offspring on a weekly basis. The eggs fertilize and develop into transparent embryos outside the mother, which allows real-time observation of organogenesis occurring inside the embryo. The embryos are small enough $(<1 \mathrm{~mm}$ in diameter) to be easily distributed and maintained in 96-well plates, which facilitates drug-screening approaches using only small quantities of drug solution. In addition, embryos are permeable to many small molecules allowing easy administration of drugs. ${ }^{8}$ Embryonic development in zebrafish is rapid and takes only few days with the majority of organs such as brain, heart, liver, intestine, and eye developing
School of Pharmacy, University of Tasmania, Hobart, TAS, Australia

Correspondence: N Gueven, School of Pharmacy, University of Tasmania, Hobart, TAS 7001, Australia Tel: + 613 62261715; Fax: +61362262870. E-mail: Nuri.Guven@ utas.edu.au

Received: 8 November 2013 Accepted in revised form: 15 January 2014 Published online: 7 February 2014 
within $24 \mathrm{~h}$ and becoming functional within a week. The free-swimming larvae hatch from around $72 \mathrm{~h}$ post fertilization (hpf) and soon start foraging for food. All of these attributes explain the steady increase in the use of zebrafish in pharmaceutical research. The large number of embryos produced per clutch makes zebrafish a prominent model for higher-throughput screening of small molecules, ${ }^{9}$ which is mandatory for preclinical drug development and toxicity assessment. ${ }^{10}$ So far, zebrafish have been used to model a wide variety of human diseases, including hereditary muscle diseases, ${ }^{11}$ neurological disorders, ${ }^{12}$ tuberculosis, ${ }^{13}$ cancer, ${ }^{14}$ cardiovascular diseases, ${ }^{15}$ and haematopoietic and infectious diseases. ${ }^{16}$ In addition, zebrafish are a valuable vertebrate model for studying vision-related disorders. ${ }^{17}$ However, there are few differences between the visual system of zebrafish and humans, which need to be taken into account.

\section{The visual system of zebrafish}

\section{Eye structure}

The visual system of zebrafish is fundamentally similar to human subjects but exhibits some notable structural differences (Figure 1, Table 1). ${ }^{17-27}$ The lens in zebrafish eyes is spheroid and not ellipsoid as compared with the human eye, and as a consequence, the zebrafish eye has a much lower volume of vitreous compared with the human eye (Figure 1a).

The zebrafish retina. As zebrafish use vision to protect themselves against predators and depend on light to search their food, their visual system develops rapidly. ${ }^{23}$ The retinal structure starts to develop from $32 \mathrm{hpf}$ and continues to develop extraordinarily fast within 5 days post fertilization (dpf). ${ }^{17}$ By then, the zebrafish retina becomes functional. Unlike the mammalian eye, that contains a specialized area within the retina that is responsible for high acuity vision, ${ }^{19}$ this so called macula is notably absent in zebrafish (Figure 1a). Comparable to the human eye, the retinal structure of zebrafish is comprised of five distinct layers (Figure 1b); three nuclear layers-the outer nuclear layer (ONL), the inner nuclear layer (INL), and the ganglion cell layer (GCL); and two plexiform layers (PL) - the inner (IPL) and the outer plexiform layer (OPL). ${ }^{19}$ The ONL consists of the cell bodies of photoreceptors (rods and cones), whereas the cell bodies of horizontal cells, bipolar cells and amacrine cells reside within the INL. ${ }^{19}$ The GCL contains

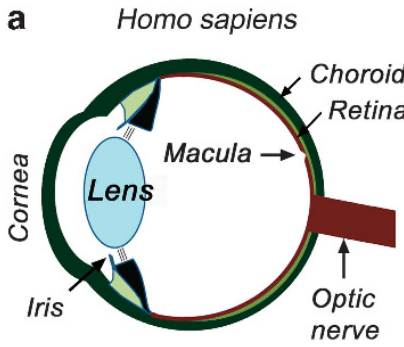

b
Homo sapiens

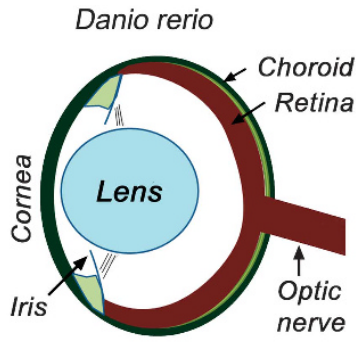

Danio rerio

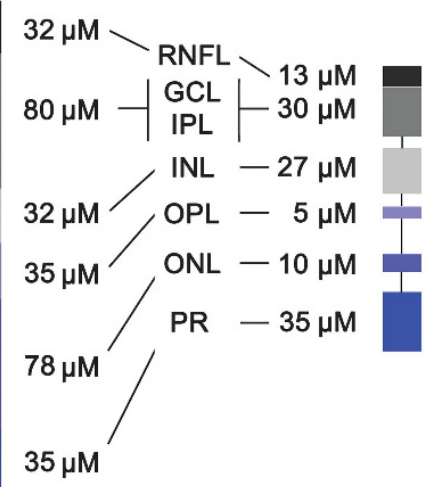

Figure 1 Comparison of the human and zebrafish eye. (a) Comparison of the human and zebrafish eye: Human and zebrafish eyes mainly differ in lens shape and space between the lens and retina. (b) Comparison of human and zebrafish retinal structure: Schematic representation of the differences of the retinal layers between human and zebrafish retina. Information on human retinal thickness taken from Yousef and Finger ${ }^{25}$ and for zebrafish derived from http://zfatlas.psu.edu/view.php?atlas=18\&s=207; zebrafish atlas, 12 months post-fertilized male transverse section. All quantifications are approximates only and may differ between individuals and areas within the retina. RNFL, retinal nerve fibre layer. 
Table 1 Comparison of the human and zebrafish eye

\begin{tabular}{|c|c|c|}
\hline Structure & Human & Zebrafish \\
\hline Eye position ${ }^{24}$ & $\begin{array}{l}\text { Frontal eyes with highly overlapped binocular } \\
\text { vision }\end{array}$ & Lateral eyes with less overlapped binocular vision \\
\hline Lens & Ellipsoidal & $\begin{array}{l}\text { Completely spherical extending partially through } \\
\text { iris providing wide-angle view }\end{array}$ \\
\hline Retina (thickness) ${ }^{25}$ & $\asymp 289 \mu \mathrm{m}$ & $\asymp 180 \mu \mathrm{m}$ \\
\hline Ganglion cells ${ }^{20}$ & Highly populated & Less densely populated \\
\hline Presence of fovea ${ }^{24}$ & Yes & No \\
\hline Vision $^{23}$ & $\begin{array}{l}\text { Cone dominant and trichromatic vision (lacks } \\
\text { UV-sensitive colour vision }\end{array}$ & $\begin{array}{l}\text { Cone dominant and tetrachromatic vision } \\
\text { (contains UV-sensitive short single cones) }\end{array}$ \\
\hline Retinotectal projection ${ }^{24}$ & $\begin{array}{l}\text { Half of the optic fibres from each eye project on to } \\
\text { the same side of the brain and other half crosses } \\
\text { over at the optic chiasm and projects to the other } \\
\text { side of the brain }\end{array}$ & $\begin{array}{l}\text { All of the optic fibres coming from each eye crosses } \\
\text { over at the optic chiasm and extends on to the } \\
\text { opposite side of the brain }\end{array}$ \\
\hline Myelination ${ }^{18,26,27}$ & $\begin{array}{l}\text { The part of optic fibres before the lamina cribrosa } \\
\text { are not myelinated, whereas fibres after protruding } \\
\text { from lamina cribrosa are all myelinated }\end{array}$ & $\begin{array}{l}\text { Whole optic fibres are myelinated. Loose, } \\
\text { single-layer myelin sheath is present around the } \\
\text { intra-retinal axon and optic nerve consists of } \\
\text { compact myelin }\end{array}$ \\
\hline
\end{tabular}

the cell bodies of ganglion cells while their axons are located in the retinal nerve fibre layer before becoming bundled into the optic nerve to carry the visual information from the eye to the brain. Synapsis between different neurons takes place at PLs. ${ }^{19}$ The synapsis between photoreceptors and bipolar cells (and horizontal cells) occurs in the OPL, and the synapsis between bipolar and ganglion cells (and amacrine cells) occurs in the IPL. ${ }^{19}$ Horizontal and amacrine cells initiate sidewise interaction in the OPL and IPL, respectively. ${ }^{28}$ Horizontal cells are responsible for enhancing contrast while amacrine cells, in addition to detecting change in illumination, also process movement and direction of light on the retina. ${ }^{28}$ In contrast to human ganglion cells where the retinal part of the axon is unmyelinated and the axon in the optic nerve is myelinated, in zebrafish the whole axon is myelinated to different extents. ${ }^{26,27}$

The photoreceptor outer segment (POS) of zebrafish consists of rods and cones, comparable to the human retina. Anatomically cones are arranged in a mosaic pattern that can be categorized into four types: short single cones (SSCs) - ultraviolet (UV)-sensitive cones, long single cones (LSCs)—blue-sensitive cones, and double cones (DCs) consisting of short (green sensitive) and long (red sensitive) cones. ${ }^{21,29}$ They are also classified based on the peak sensitivities of the photopigment present in each cone as follows: UV (360-361 nm), short (S, 407-417 nm), medium $(\mathrm{M}, 473-480 \mathrm{~nm})$, and long (L, 556-564 nm) cones. ${ }^{21}$ Based on the four cone types in zebrafish, their vision is tetrachromatic. In contrast, the human eye lacks the UV-sensitive cones and therefore has only trichromatic vision. ${ }^{23}$ Unlike cones that have four types of opsins as their photopigment, the rods consist of only a single type and contain rhodopsin as their photopigment. ${ }^{23}$
During the development of the zebrafish retina, the SSCs become distinct at 5-6 dpf while LSCs appear at 7-8 dpf and the DCs at 10-12 dpf. However, all of the visual pigments are expressed between 50 and $55 \mathrm{hpf}$, so the various cone types are presumably there at 50-55 hpf but not recognizable. The first rod cells appear at 5-6 dpf. ${ }^{17,30}$ Although small rod responses can be detected at this stage, full rod responses are not evident until 15-21 dpf. ${ }^{17,31,32}$ Therefore, until $15 \mathrm{dpf}$ zebrafish only have functional cones that are responsible for colour vision.

\section{Visual processing}

Light signals entering the eye are first refracted from the cornea to the lens and then projected onto the retina. The signal is then collected by the photopigment of the photoreceptor layer where the rod and cone cells convert the light signals into electrical signals ${ }^{33}$ that are transmitted via the horizontal, amacrine, and bipolar cells to the RGCs. ${ }^{28}$ RGCs give rise to the nerve fiber layer, which is responsible for carrying the signals from the eye, via the optic nerve to the optical centres in the brain.

\section{Eye development}

The initial development of the eye structures in zebrafish generally resembles those of other vertebrates ${ }^{34,35}$ (Figure 2). Eye morphogenesis begins with the evagination of the optic vesicle from the forebrain at $11 \mathrm{hpf}$ as a flat structure, which then extends laterally, forming an optic lobe. ${ }^{34,36}$ The anterior portion of the optic lobe remains attached to the forebrain through the optic stalk. ${ }^{36}$ The optic vesicle finally gives rise to the neural retina and pigmented epithelium at $15 \mathrm{hpf}$. 


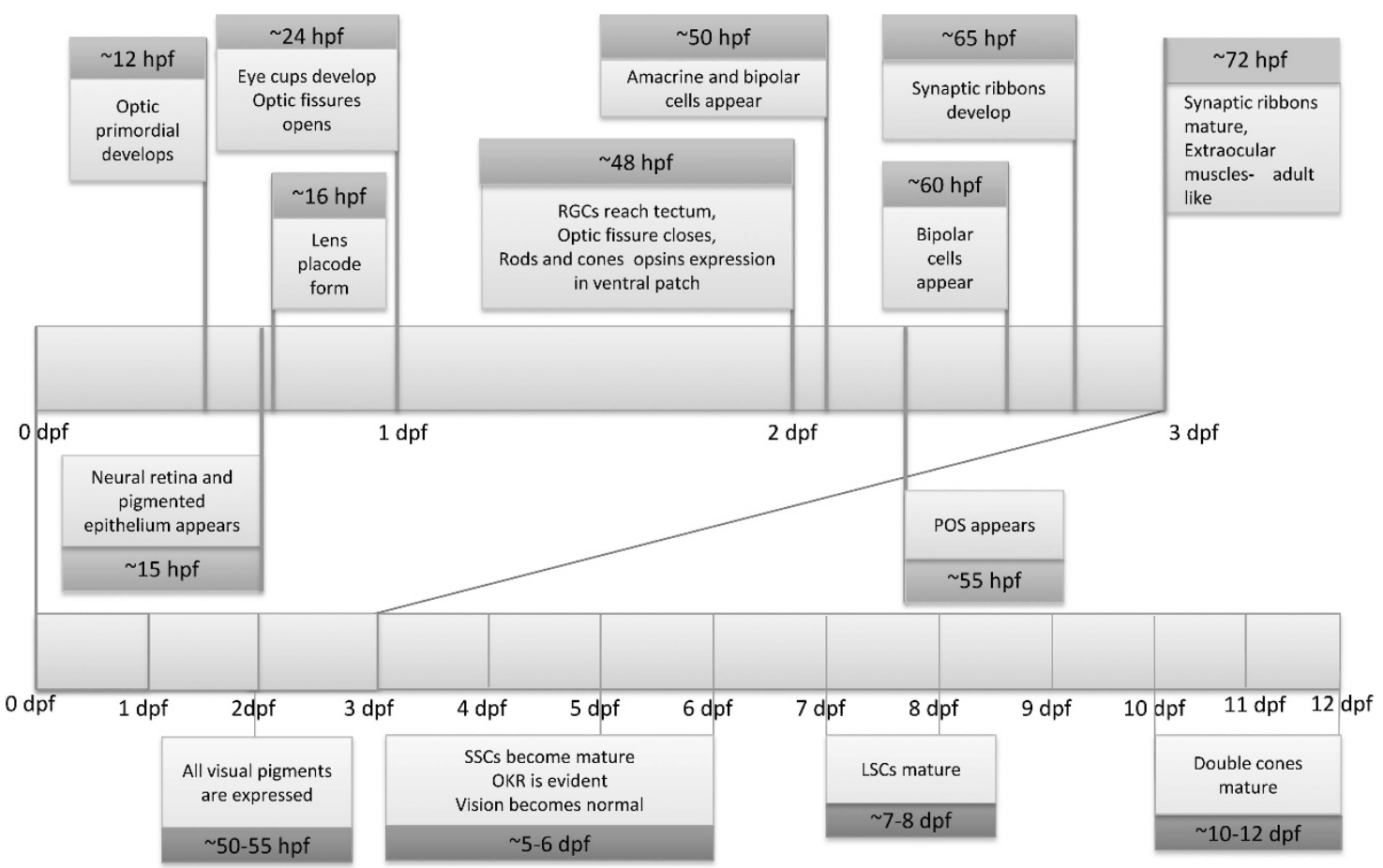

Figure 2 Sequences of the eye development in zebrafish: dpf, days post fertilization; hpf, hours post fertilization; LSC, long single cone; OKR, optokinetic response; POS, photoreceptor outer segment; RGC, retinal ganglion cell; SSC, short single cone.

By 16-20 hpf, the eyecups are developed through a series of different stages from the optic lobe. Surface ectoderm cells underlying the eyecups thicken and form a lens placode at around $16 \mathrm{hpf}$. Delamination of the lens placode from the surface ectoderm occurs at approximately $24 \mathrm{hpf}$ and then detaches fully by $26 \mathrm{hpf}$. By $30 \mathrm{hpf}$, the corneal epithelium is formed from the surface ectoderm, which overlies the lens. Further morphogenesis of the eyecups forms an optic fissure by $24 \mathrm{hpf}$ and closes thereafter at $48 \mathrm{hpf}$, which signals that morphogenesis of the embryonic eye is largely terminated and that only retinal neurogenesis is in progress at that time. ${ }^{7,34,35}$ At around $32 \mathrm{hpf}$, the first ganglionic cells differentiate ${ }^{37}$ and soon after that their axons reach the optic tectum. ${ }^{19}$ The amacrine and horizontal cells within the INL first appear at $50 \mathrm{hpf}$, and by $55 \mathrm{hpf}$ rod and cone outer segment in the ONL are developed. ${ }^{35}$ Rods cells and Muller glial cells are the last to differentiate. ${ }^{38}$ The presynaptic photoreceptor ribbon (Figure 3) develops at about 65 hpf. ${ }^{35}$ Visual information is processed once the ribbon synapses of the bipolar cells reach maturity at $74 \mathrm{hpf}, 35$ which are responsible for passing the light-induced changes in photoreceptor cell membrane potential down the retinal circuit. ${ }^{39}$ This means that the retinal structure becomes functional at around 74 hpf. $^{40}$ At the same time, the extra-ocular muscles become fully functional, which is a prerequisite for tracking of moving objects by eye movement. ${ }^{41}$
Consequently, visually mediated response improves thereafter with adult-like performance after $96 \mathrm{hpf}$, where full tracking of eye movement (optokinetic response (OKR)) is evident.

\section{Why zebrafish are a good animal model for ophthalmological studies}

Unlike mouse models, zebrafish are relatively easy to maintain at a fraction of the cost. Zebrafish are prolific breeders and produce as many as 100-200 eggs on every mating, while a pair of mice reliably produces a maximum 6-8 offspring. This large number of eggs per clutch in zebrafish allows higher-throughput screening for the identification of mutants and potential drug candidates. ${ }^{17}$ In many respects, the zebrafish's visual system mirrors the human situation better compared with other animal models. Contrary to mice that have rod-dominated vision, zebrafish have cone-dominant vision like humans, ${ }^{7}$ which is a prerequisite to study human disorders associated with cone degeneration, such as AMD. ${ }^{22}$ In addition, the eyes of zebrafish develop fast from only $12 \mathrm{hpf}$ and display a functional visual system by $5 \mathrm{dpf}$. This is significantly faster compared with mice (around 15-20 days) ${ }^{42,43}$ and allows to study visual function already in 5-day-old larvae. Furthermore, the significant amount of genetic information available from zebrafish mutants associated with defective visual 


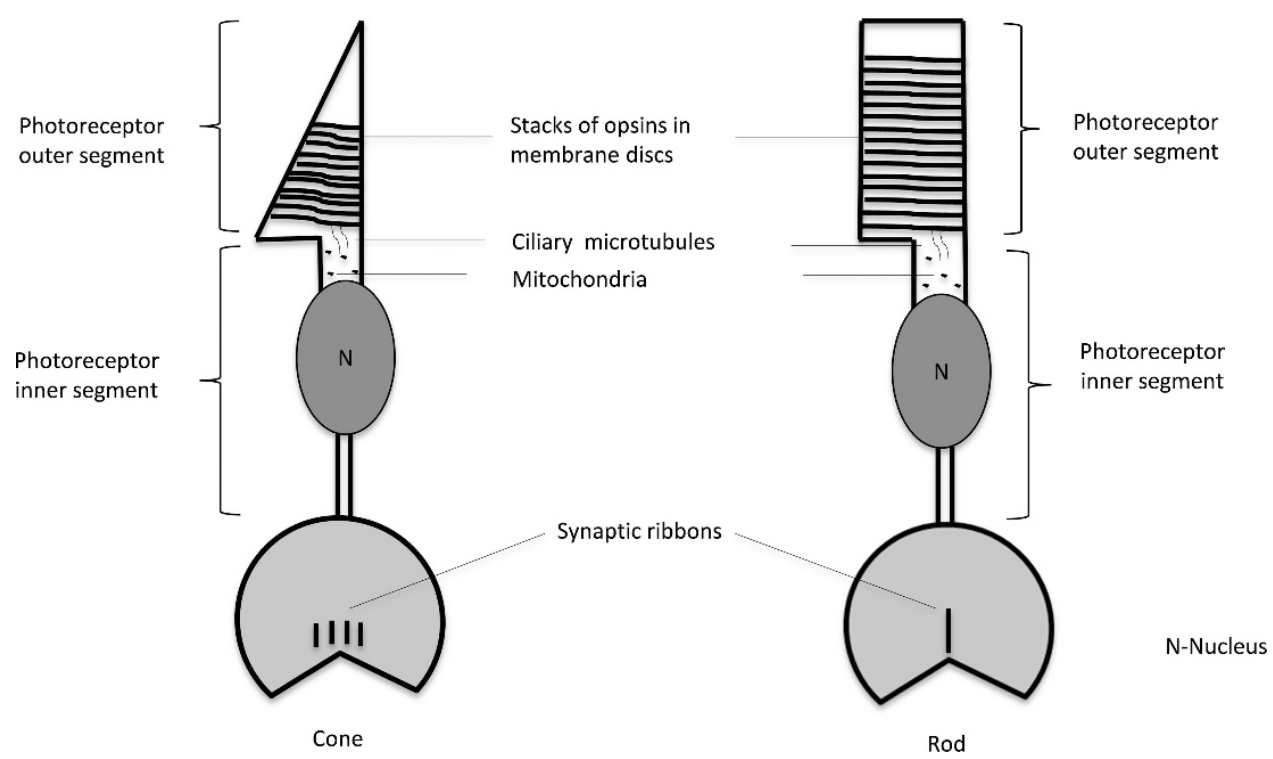

Figure 3 Synaptic ribbons: Photoreceptors showing presynaptic ribbons, which are responsible for signal transmission from photoreceptor layer to bipolar and horizontal cells.

development and function illustrates the power of this model for understanding human ophthalmological disorders.

\section{Measurement of visual behaviour}

Behavioural responses have been utilized as an important tool for screening genetic defects of the visual system as well as to study the development of the visual system in zebrafish. Several well-described behavioural responses with unique advantages and limitations have been used so far (Table 2).

\section{OKR}

The OKR is based on the eye movement reflex in response to a moving stimulus to help stabilize the image on the retina to maintain visual acuity and is evidence of a fully functional visual system in zebrafish. ${ }^{44,45}$ The OKR is tracking eye movements, which consist of smooth pursuits (slow phase) and fast resetting saccades in the opposite direction. ${ }^{24}$ OKR begins at around $74 \mathrm{hpf}$ when only $5 \%$ of the zebrafish larvae respond to the visual stimulus, which then increases steadily to $100 \%$ by 80 hpf. $^{34,40}$ The OKR improves in terms of time spent in tracking the stimulus and velocity of tracking eye movement by $96 \mathrm{hpf.} .^{3,40,41}$ For this method, zebrafish larvae are immobilized to restrain the body while maintaining the ability for eye movement and are then exposed to a visual stimulus that usually consists of alternate black and white stripes that move around the larvae. 4,23,24,46 The larvae respond to this stimulus by moving their eyes in the direction of the moving stripes. ${ }^{23}$ Experimental visual stimuli, such as spatial frequency, contrast, and angular velocity can be altered in this setting to gain a deeper understanding of a potential defect. ${ }^{46}$ With some experimental adaptations, such as constant supply of oxygen-rich water while body movement is restrained, this method can be applied to adult fish as well. ${ }^{47}$ This assay is rapid and responses of larvae or adult fish can be processed within only few minutes. ${ }^{44}$ OKR was used to identify and characterize many zebrafish mutants that are associated with vision system defects (Table 3), such as bumper mutant (defect in lens development), noir, dropje, lakritz mutants (defect in retinal structure), belladonna mutant (RGCs axon misrouting), and grumpy and sleepy mutants (optic nerve disorder). ${ }^{4}$ Other examples with visual function defects are the brass mutant with a phenotype similar to glaucoma. ${ }^{48}$ In addition, OKR was also useful in isolating a colour-blind mutant by changing the colour of visual stimulus. ${ }^{49}$ In summary, the OKR appears to result in more a robust, reliable, and quantifiable behavioural data compared with other methods described below, especially after automated commercial systems have become available. ${ }^{3,49}$

\section{Optomotor response (OMR)}

The OMR tests the visual behaviour of zebrafish by their tendency to swim towards moving black and white stripes. ${ }^{6}$ As numerous fish with possible visual function defects can be tested at once, this OMR assay enables higher-throughput analysis compared with the OKR. 
Table 2 Summary of behavioural assays used in zebrafish

\begin{tabular}{|c|c|c|c|c|}
\hline Assays & Suitability & Validity & Advantages & Disadvantages \\
\hline $\begin{array}{l}\mathrm{SR}^{40,53} \\
\mathrm{VMR}^{55,56}\end{array}$ & $\begin{array}{l}\geq 4 \mathrm{dpf} \\
\text { (embryo) }\end{array}$ & Yes & $\begin{array}{l}\text { Suitable for analysing simple visual function, } \\
\text { such as differentiating light from darkness } \\
\text { Useful to study visual function development } \\
\text { VMR complements OKR and is useful for } \\
\text { visual mutants screening }\end{array}$ & $\begin{array}{l}\text { Not suitable for larvae } \geq 5 \mathrm{dpf} \text { as spontaneous } \\
\text { movement is often difficult to distinguish from } \\
\text { startle response } \\
\text { Not suitable for screening visual defect mutants }\end{array}$ \\
\hline $\mathrm{OKR}^{4,6,41,46}$ & $\begin{array}{l}5-7 \text { dpf } \\
\text { (embryo } \\
\text { and adult) }\end{array}$ & Yes & $\begin{array}{l}\text { Suitable for complex visual function test } \\
\text { (visual acuity, contrast sensitivity, colour } \\
\text { blindness, etc) } \\
\text { Useful for mutant screens. Provides specificity } \\
\text { in screening mutants }\end{array}$ & $\begin{array}{l}\text { Time consuming assay (test one fish at a time) } \\
\text { Possibility of missing the strong mutants.- } \\
\text { randomly selected }\end{array}$ \\
\hline $\mathrm{OMR}^{4,6,50}$ & $\begin{array}{l}>6 \mathrm{dpf} \\
(\mathrm{embryo} \\
\text { and adult) }\end{array}$ & Yes & $\begin{array}{l}\text { Fast assay for high-throughput analysis of } \\
\text { visual mutants (large number of fish can be } \\
\text { analyzed at once) } \\
\text { Lower risk of missing mutants while screening }\end{array}$ & $\begin{array}{l}\text { Lack of specificity in result (possibility of false } \\
\text { result sometimes)-as it does not test each and } \\
\text { every individual fish }\end{array}$ \\
\hline $\mathrm{PTB}^{3}$ & $\begin{array}{l}>6 \text { dpf } \\
\text { (embryo } \\
\text { and adult) }\end{array}$ & No & Suitable for simple light responses & $\begin{array}{l}\text { Not suitable for mutants screening-due to } \\
\text { unreliable result }\end{array}$ \\
\hline $\mathrm{ER}^{57,59}$ & $\begin{array}{l}>2 \text { months } \\
\text { (adult) }\end{array}$ & Yes & $\begin{array}{l}\text { Simple, robust method } \\
\text { Provides high-throughput analysis } \\
\text { Useful in screening visual mutants, visual } \\
\text { sensitivities as well as for drug identification } \\
\text { and toxicity studies }\end{array}$ & \\
\hline
\end{tabular}

Abbreviations: dpf, days post fertilization; ER, escape response; OKR, optokinetic response; OMR, optomotor response; PTB, phototactic behaviour; $\mathrm{SR}$, startle response; VMR, visual motor response.

Table 3 Summary of existing zebrafish model of some human ocular diseases

\begin{tabular}{|c|c|c|c|}
\hline Affected eye structure & Zebrafish mutant/morpholino & Human ocular disease & Assays used \\
\hline Lens ${ }^{65,71,75}$ & $\begin{array}{l}\text { Mutation of CRYGC gene Cloche } \\
\text { Mutation of PITX3 and FOXE3 }\end{array}$ & Congenital cataract & Histology \\
\hline Anterior segment ${ }^{80,83}$ & $\begin{array}{l}\text { Mutation of FOXC1 } \\
\text { Bugeye mutant }\end{array}$ & Glaucoma & $\begin{array}{l}\text { Immunohistological } \\
\text { OMR, electroretinograms, histology }\end{array}$ \\
\hline Retina & Oval (Ovl), elipsa (elip) mutants & $\begin{array}{l}\text { Human ciliopathies (oculo-renal } \\
\text { diseases)-Bardet-Biedl syndrome, } \\
\text { Senior-Loken syndrome }\end{array}$ & OKR, electroretinograms, histology \\
\hline Photoreceptors ${ }^{105}$ & REP 1 gene mutation & $\begin{array}{l}\text { Oculo-cutaneous albinism- } \\
\text { human choroideremia }\end{array}$ & $\begin{array}{l}\text { SR, transmission electron } \\
\text { microscopy }\end{array}$ \\
\hline $\mathrm{RPE}^{108,111}$ & Fade out (fad) mutant & Hermansky Pudlak syndrome & $\begin{array}{l}\text { Histology, transmission electron } \\
\text { microscopy, immunohistochemistry }\end{array}$ \\
\hline Retinal & Fli-EGFP-Tg Transgenic & Diabetes retinopathy, & Confocal microscopy \\
\hline Vasculature $^{88-90}$ & gnn mutant & $\begin{array}{l}\text { AMD } \\
\text { AMD }\end{array}$ & $\begin{array}{l}\text { OKR, histology, } \\
\text { immunocytochemistry, electron } \\
\text { microscopy }\end{array}$ \\
\hline Retinotectal projection ${ }^{4}$ & Belladonna (bel) mutant & Human congenital nystagmus & OKR, histology, electroretinograms \\
\hline
\end{tabular}

Abbreviations: AMD, age-related macular degeneration; OKR, optokinetic response; OMR, optomotor response; RPE, retinal pigmented epithelium; $\mathrm{SR}$, startle response.

However, it has to be noted that compared with the OKR there is a significantly higher risk to miss mutants when using OMR. ${ }^{6}$ The experimental setup to measure the OMR is similar to that of the OKR except that the embryos or adult fish are not constrained and are able to swim towards the perceived black-white stripes. ${ }^{50}$ This type of behavioural response shown by zebrafish is one of the innate behaviours known as taxis where fish orient themselves in the direction of the stimulus. ${ }^{51}$ This behaviour is important to maintain their orientation in water. ${ }^{51}$ In the assay, the wild-type fish swim towards the perceived motion and gather at one end of a chamber while zebrafish with visual defects swim in random patterns. ${ }^{50} \mathrm{OMR}$ is suitable to test the visual acuity of both larvae and adult zebrafish and has been used to isolate at least 17 mutants from 411 previously identified loci in zebrafish that affect visual function so far. $4,17,40,50$ Furthermore, the development of visual behaviour in zebrafish under different light conditions $(\mathrm{ON} \text { and } \mathrm{OFF})^{50}$ and the effects of ethanol 
exposure on embryonic eye development ${ }^{50,52}$ have also been studied using OMR.

\section{The startle response (SR)}

The SR in zebrafish is based on a form of body movement within $2 \mathrm{~s}$ in response to a sudden exposure to a low intensity light $\left(\sim 60 \mu \mathrm{W} / \mathrm{m}^{2}\right)$ or an acoustic signal and is also useful to determine the development and maturation of the visual system in zebrafish. ${ }^{40,53}$ The SR is typically first seen at $68 \mathrm{hpf}$ where $17 \%$ of the fish show SRs, which then steadily increase to $100 \%$ at $79 \mathrm{hpf}$ at a time when the POS appear abundantly together with a functional retina and fully developed synaptic ribbon. ${ }^{40}$ This assay has been successfully used to assess the visual development in zebrafish embryos as well as used to detect defects of visual development in response to exposure to toxins, such as methylmercury. ${ }^{54}$ Recently, a modification of the SR, called visual motor response (VMR), was described, which can be performed on $4 \mathrm{dpf}$ zebrafish larvae to screen for visual mutants. VMR was developed to confirm the result of OKR and examines whether visual mutants that fail to detect motion under OKR can still detect changes in light intensities. ${ }^{55,56}$

\section{Phototactic behaviour (PTB)}

The PTB is based on the tendency of zebrafish with normal vision to move towards an illuminated chamber. ${ }^{3}$ The experimental setup for this assay consists of a rectangular acrylic box with two chambers inside separated by a sliding bar. There are two methods in which PTB can be assessed. ${ }^{3}$ The first approach is based on two chambers, which are equally illuminated. The fish are allowed to distribute between both chambers, and the number of larvae in each chamber are counted after a set of time interval. Then the same procedure is repeated with only one chamber illuminated, while the other chamber is covered. In general, larvae between 7-14 dpf are suitable for the first method. For the second method, larvae aged between 7 and 19 days are kept in a darkened chamber for up to 2 min before the partition is removed, and the fish are free to enter the second illuminated chamber. The partition is replaced after few minutes, and the numbers of fish in both chambers are quantified. Typically, fish with normal vision will move towards the illuminated chamber, whereas fish with vision defects will not show a preference for the illuminated chamber. For this assay, it was noted that fish move towards the illuminated chamber in both methods, which is evidence of PTB in zebrafish. However, there appears to be no significant difference in the number of fish moving from one chamber to another in the light and dark condition approach, which has to be taken into account when interpreting the results. Because of this limitation, the PTB is less suitable for screening zebrafish for mutants with defective vision.

\section{Escape response (ER)}

Another method to assess vision in zebrafish is the ER, which is based on the natural tendency of zebrafish to evade an approaching predator. In a typical experimental setup, zebrafish are kept inside a white, circular rotating drum with a single black strip that mimics a threatening predator. ${ }^{57}$ In this assay, zebrafish show a tendency to keep on opposite site of the black stripe. The response of zebrafish either to move towards the visual cues or to move away from it is determined by the size of the visual cue. ${ }^{58}$ Zebrafish orient themselves towards a small moving visual stimulus as in the case of OMR. As the size of the visual stimulus increases, their behavioural response change into aversive turns, and they move away from it as in the case of ER. This is well explained as a transition from a prey capture-related orienting response to a predator-avoidance response. ${ }^{58}$ This test is robust and has been used before in several studies to characterize mutants that affect vision, ${ }^{57,59}$ such as $n b a$ (night blindness $a$ ) and $n b b$ (night blindness $b$ ), which are dominant mutants with adult retinal degeneration. ${ }^{57}$ Using ER, the role of the circadian clock in modulating visual sensitivity has also been studied in zebrafish. ${ }^{60,61}$ When the threshold light intensity to initiate an ER was measured over a period of $24 \mathrm{~h}$ under light dark cycle, the threshold light intensity was at its highest before 'lights on' early in the morning and at its lowest before 'light off' in the late afternoon. Therefore, the authors concluded that zebrafish are more sensitive towards visual stimuli at dusk than at dawn. ${ }^{60}$

\section{Limitations of visual behaviour-based assays}

It has to be pointed out that the assessment of visual function in zebrafish by the assays described above is entirely based on their behavioural response to the visual stimulus. As a consequence, there is a potential that these assays could produce misleading results. In particular, it can be hypothesized that defects in tissues other than the eye that are nevertheless crucial for behaviour, such as neuronal circuits that affect muscle function, have the potential to produce misleading results. Genetic analysis of visual behaviour in zebrafish mutants (10 loci, 12 new alleles) did reveal defective visual behaviours (OMR, OKR), with no obvious morphological defect in retina and RGC projection. ${ }^{6}$ This study has highlighted the potential involvement of brain functions beyond retinotectal projection, which is not unexpected given our 
knowledge of drugs that can alter behaviour. For example, it has been reported that acute alcohol exposure reduces the fear responses and zebrafish become unresponsive towards visual cues. ${ }^{62}$

Nevertheless, some drugs can inhibit motility, thus ending up with false impression on visual behaviour assays that is examined by the fish movement such as $\mathrm{OMR}$, therefore further assessment in such condition is necessary. ${ }^{63}$

Finally, defects in all processes and systems that are involved from the processing of visual information to the actual observed change of visual behaviour, such as motor function, have to be seen as possible sites that could influence results. As such, processing of the visual signal in the visual cortex (in higher vertebrates) or optic tectum (in case of zebrafish) only serves as a prerequisite for induced behaviour, therefore dysfunction not only in the visual cortex but also other brain areas involved in decision-making could potentially lead to unresponsiveness to the visual stimulation. Thus, confirmation of behavioural assay results with additional methods, such as detection of pathology in the retina or optic nerve or the use of electroretinography ${ }^{5,57}$ will significantly increase the reliability of results.

\section{Modelling human eye diseases in zebrafish}

The strength of the zebrafish model lies in the ability to potentially screen thousands of compounds and drugs for their therapeutic potential in human diseases. Many human pathological conditions from Alzheimer's disease to metabolic syndrome have been successfully modelled in zebrafish. Over the past decade, zebrafish have also been used to model several human ocular diseases, such as cataract, glaucoma, DR, and AMD (Table 3).

\section{Cataracts}

Globally, cataracts are the leading cause of blindness ${ }^{1}$ caused by opacity of the lens. ${ }^{64}$ Cataracts are most prevalent in the aged population but also occur as congenital cataracts in children with genetic predisposition in the first year of life. ${ }^{64}$ Some studies have described zebrafish as a promising animal model to study dominant congenital cataracts. ${ }^{65,66}$ Under normal conditions, the protein crystalline ( $\beta \gamma, \gamma \mathrm{C}$ types) assures lens and cornea transparency and is also necessary for refractive power. ${ }^{67,68}$ Therefore, it is expected that mutations in the crystalline gene are linked to cataract formation in humans. ${ }^{69,70}$ Recently, a cataract-causing gene has been identified using a zebrafish animal model. Mutation of the CRYBA2 gene, a member of $\beta \gamma$ crystalline causes congenital cataracts. ${ }^{66}$ Furthermore, mutation in
CRYGC gene ( $\gamma$ C-crystalline) makes the lens less thermally stable and increases the risk of lens opacity when exposed to heat and UV radiation and ultimately also leads to cataract formation. ${ }^{65}$ Similarly the cloche zebrafish mutant displays cataracts associated with a defect in gamma crystalline induced by alpha A crystalline. ${ }^{71}$ Alpha A crystalline is essential for decreasing insolubility of gamma crystalline, which in turn increases lens transparency and initiates the differentiation of lens fibre cells. Therefore, this study has highlighted that cataract development could be preventable, provided that a sufficient alpha A crystalline expression can be achieved in the lens. Human congenital cataracts have also been modelled in zebrafish by gene knockdown experiments. For example, mutations in the PITX3 and FOXE3 genes, which are expressed in the lens epithelium, cause anterior segment defects, including cataracts. ${ }^{72-75}$

\section{Glaucoma}

Glaucoma is the second leading cause of blindness in the world. ${ }^{1}$ Glaucoma is an array of adult onset retinal neuropathies characterized by progressive degeneration of RGCs and the optic nerve head. ${ }^{7}$ Despite the anatomical difference of trabecular meshwork ${ }^{76}$ and aqueous humour dynamics in human and zebrafish, ${ }^{77}$ the overall similarities in aqueous humour outflow tissue structure and average intraocular pressure $(\mathrm{IOP})^{48}$ make zebrafish a potential model to study the complex genetics of glaucoma. Severe myopia, anterior segment defects (iris, trabecular meshwork, and cornea), and increased IOP, which precedes blockage of aqueous humour drainage, are the major risk factors associated with glaucoma. ${ }^{78}$ The disease-risk phenotype of glaucoma is well studied in zebrafish mutants and was successfully modelled in zebrafish, for example, by mutation of the FOXC1 (forkhead transcription factor) gene. ${ }^{79-81}$ FOXC1 is expressed in anterior segment and periocular mesenchymal cells ${ }^{82}$ and is essential for the development and maintenance of the anterior segment of the eye. Therefore, mutations in this transcription factor cause a glaucoma-like pathology, such as abnormalities in the iris, trabecular meshwork, and cornea. Furthermore, the mutation of the gene coding for low-density lipoprotein receptor-related protein 2 represented by the bugeye mutant of zebrafish has recently been analyzed with regards to glaucoma-linked risk factors using OMR, electroretinograms (ERGs) and histology analysis. 83,84 This bugeye mutant showed glaucoma-risk phenotypes such as enlarged eyes as a result of increased IOP, decrease in retinal ganglion cell numbers at 3 months, and outer retinal dysfunction by 5 months due to prolonged mechanical stress. 


\section{AMD}

AMD is a degenerative disease of the central retina and the third leading cause of blindness worldwide mostly affecting people aged $>50$ years. ${ }^{1,85}$ It is characterized by a presence of pale yellowish lesion in macula and peripheral retina as a result of deposition of acellular polymorphous debris called drusen. The pathology includes the degeneration of cone cells, RPE, and Bruch's membrane. Later stages show choroid neovascularization, ${ }^{86}$ which subsequently leads to a loss of colour vision and visual acuity. Zebrafish have been used to study retinopathy (seen in AMD and DR) induced by hypoxia. ${ }^{87}$ Hypoxia is induced in adult zebrafish by exposing them to hypoxic water for 3-10 days, ${ }^{88}$ which then initiates neovascularization and produces immature, leaky, and disorganized blood vessels. ${ }^{89}$ The use of orally active anti-vascular endothelial growth factor (anti-VEGF) demonstrated significant efficacy against the pathological symptoms in zebrafish. ${ }^{88,89}$ These findings suggest that VEGF-induced hypoxia is an essential component of retinal neovascularization in AMD and DR. ${ }^{88}$ Similarly, the zebrafish mutant gnn displays a phenotype analogous to AMD in humans. The macroscopic and behavioural screening of gnn zebrafish mutant from 2 to 9 days revealed dystrophy of red cones at around $5 \mathrm{dpf}$, which later spread to all other cone types, ${ }^{90}$ a pathology that is reminiscent of AMD in human patients.

\section{DR}

Diabetes mellitus (DM) affected 366 million people globally in 2011, which is estimated to increase to 552 million in $2030 .{ }^{91}$ Around $80 \%$ of DM patients develop DR within 20 years of being diagnosed with DM. ${ }^{92}$ DR is a frequent pathology as a result of microvascular complications of DM that affect the retinal structure. ${ }^{93}$ The risk factors for DR include neovascularization, vitreous haemorrhage, loss of blood-brain barrier (BBB) integrity, and macular oedema. ${ }^{94}$ Retinal neovascularization in DR generally tends to be more fragile and leaky, which leads to vitreous haemorrhage that ultimately leads to vision loss. ${ }^{94}$ The current treatment options for DR are limited to symptomatic relief and are not able to reverse the structural abnormalities once the disease has progressed. Therefore, animal models have been used to provide insight into underlying pathogenesis of DR and to screen novel drugs entities. There is a growing interest in the use of zebrafish in DR due to the fact that they exhibit similar retinal vascular physiology and pathology compared with mammals. ${ }^{95}$ Hyperglycaemic zebrafish are shown to have similar retinal structure abnormalities as seen in diabetic patients. ${ }^{96}$ Hyperglycaemia can be induced in adult zebrafish by immersing alternately in glucose solution and water over 28 days followed by retinal examination. IPL and INL layers were shown to be significantly reduced in treated zebrafish, with the thickness of the IPL approximately $55 \%$ of the INL. ${ }^{96}$ This study provided some support to the idea that zebrafish can be utilized to model DR. This finding was confirmed by another study where visual impairment was evident after inducing hyperglycaemia in zebrafish. ${ }^{97}$ In this study, hyperglycaemia affected the cone photoreceptor neuron layer as shown by their structural degeneration and through the use of ERGs. ${ }^{97}$ This study suggested that neuro-protective agents in combination with the drugs to combat the vascular complications would provide clinical benefits in DR. Furthermore, hypoxia-induced retinal neovascularization or angiogenesis in the adult Fli-EGFP-Tg transgenic zebrafish model recapitulates DR in humans. Using these models, it is a realistic prospect to assess the therapeutic efficacy of anti-angiogenic agents. 88,89

\section{Human congenital nystagmus (HCN)/infantile nystagmus syndrome (INS)}

INS is a congenital occulomotor disorder and genetically heterogeneous condition having different modes of inheritance: X-linked, autosomal dominant, and autosomal recessive. ${ }^{98} \mathrm{HCN} / \mathrm{INS}$ is characterized by periodic alternation of involuntary oscillations of the eye consisting of slow movement of the eye called slow phase followed by quick phase to focus on the object of interest called saccades. The pathophysiology of the disease is poorly understood. ${ }^{99}$ Studies have shown that three autosomal (NYS2, NYS3, and NYS4) and X-chromosomal loci (NYS1, NYS5) are thought to have a key role in aetiology of congenital nystagmus. Recently, it was shown that mutation of the FRMD7 gene on loci NYS1, which is expressed in neuronal tissue of the developing retina, mid brain, and hindbrain, is associated with INS. ${ }^{98}$ INS has been modelled in zebrafish. ${ }^{4,24}$ A large number of homozygous belladonna (bel) mutants were found to display a reversed OKR indicative of a projection defect. In contrast to wild-type animals, visual stimulation of one eye in bel mutants leads to movement of unstimulated eye, which is indicative of optic nerve misrouting owing to absence of optic chiasm. These findings in zebrafish are in good agreement with a recent study that concluded that optic nerve misrouting could be a primary cause of INS. ${ }^{100}$

\section{Human ciliopathies}

Human cilliopathies are caused by a diverse set of mutations that lead to defects in a range of cilia-containing 
tissues, ${ }^{101}$ and retinal dysfunction is frequently associated with ciliopathies. The photoreceptors consists of mainly two segments: the outer segment (POS) contains stacks of membranous discs in which photopigments are embedded and the inner segment contains the nuclei and the machinery necessary for synthesis of proteins and metabolites. ${ }^{102}$ These two segments are linked by a connecting cilium that contains microtubules. ${ }^{102}$ Photopigments are synthesized in the inner segment and transported to the POS by the ciliary microtubules (Figure 3), a mechanism that is known as intraflagellar transport (IFT). ${ }^{103}$ Around $10 \%$ of the POS is replaced every day. For this purpose, proteins are transported from the inner segment by IFT to ensure the maintenance of the POS. ${ }^{104}$ Consequently, mutations of IFT proteins (present in the flagella of photoreceptor cells) have been shown to lead to retinal degeneration and subsequent blindness such as in Bardet-Biedl syndrome and Senior-Loken syndrome that are characterized by photoreceptor dysfunction and renal dysplasia. ${ }^{103,104}$ These human ciliopathies are mirrored in the zebrafish mutants oval (ovl) and elipsa (elip) that show outer retinal dystrophy and renal dysfunction. ${ }^{39,105}$

Analysis of the visual defect in the ovl and elip mutants using OKR (high-contrast visual stimulation), electroretinography, and histology revealed involuntary eye movements as well as light-insensitive and progressive degeneration of the POS. ${ }^{39,105}$ Mutations in ovl affect intraflagellar components such as IFT 88, IFT 52 , and IFT 57 and, as a consequence, produces defective cilia that lead to POS degeneration and vision impairment. ${ }^{39}$ Recently, the Rer1p gene, which is highly expressed in the cilia of zebrafish and mammals has been implicated in maintaining cilliary length and sensory function. ${ }^{106}$ Therefore, but not unexpected, mutations in Rer1p caused cilliary shortening and subsequent loss of sensory function, including vision loss. ${ }^{106}$

\section{Others}

Being situated in close proximity to each other, genetic defects of the RPE often lead to subsequent dystrophy of photoreceptor cells. ${ }^{107}$ This situation is observed in human choroideremia (CHM) and Hermansky-Pudlak syndrome, which have been also well modelled in zebrafish mutants.

Human CHM. Human CHM is an inherited human retinal blindness caused by mutations of the Rab escort protein 1 ( $R E P$ 1) gene, which leads to a progressive degeneration of rod photoreceptors subsequent to pigmentation defects in RPE cells. ${ }^{108,109}$ This disease is modelled in the zebrafish larvae mutant line ru848, in which partial retinal dystrophy has been observed. ${ }^{108}$
However, it has to be noted that in humans the related gene, rep2, can compensate for the loss of REP 1. As zebrafish lack rep2 gene expression, ${ }^{110}$ the relevance of the ru848 mutation for the study of human CHM remains to be evaluated.

Hermansky-Pudlak syndrome. Hermansky-Pudlak syndrome is a group of disorders characterized by oculocutaneous albinism (decrease in pigmentation of the skin, eye, and pilar caused by defective melanin production pathway), bleeding, and lysosome-related organelle defect. ${ }^{107}$ This syndrome has been reported in the zebrafish fading vision mutant ( $f a d)$ that shows depigmentation of the RPE followed by loss of photoreceptor cells. ${ }^{111}$

\section{Ophthalmological drug discovery and development in zebrafish}

Zebrafish have been utilized to study the cellular and molecular mechanism of pathogenesis of human eye diseases. At the same time, the benefit of large clutch size of this model provides an opportunity for screening drugs that have the potential to be used for treating human eye diseases. For example, aminoglycosides (gentamicin and paromycin) have shown remarkable therapeutic efficacy in the zebrafish mutants (lamb and pax 2). ${ }^{112}$ These mutants recapitulate coloboma formation, which can be characterized by a triangular eyelid defect as a result of unsuccessful closure of choroid fissure during early morphogenesis of the eye. ${ }^{64}$ These findings reveal the potential of aminoglycosides in the treatment of human coloboma. ${ }^{112}$ Furthermore, the zebrafish model has established itself as a prominent model system for hypoxia-induced retinopathy. ${ }^{87}$ These hypoxic zebrafish models have been utilized to test oral anti-angiogenesis inhibitors (anti-VEGF agents), such as sunitinib and $z \mathrm{~m} 323881 .{ }^{89}$ These drugs rescued neovascularization of the retina confirming that VEGF, triggered by hypoxia to compensate for the reduced oxygen supply by forming new blood vessels, has a key role in retinal angiogenesis. ${ }^{89}$

\section{Drug-related ophthalmological toxicity assessment in zebrafish}

More than 200 currently used drugs are associated with ophthalmic toxicity as a result of adverse drug reaction. ${ }^{113}$ Assessment of oculotoxicity associated with drugs at the early stage of their development is crucial. However, this process is partly hindered due to a lack of predictive, convenient methods as well as the high expense associated with testing drug candidates in mammals. ${ }^{63,114}$ For many years, researchers have been 
using zebrafish assays for drug toxicity screening with a view to provide early drug safety assessments. ${ }^{115,116}$ So far, zebrafish have been used to predict drug-related cardiotoxicity, ototoxicity, developmental toxicity, neurotoxicity, oculotoxicity, and many more. ${ }^{115,116}$ Furthermore, zebrafish have efficiently predicted toxicity associated with the effects of systemic drugs in humans. ${ }^{116}$ More recently, a study described zebrafish as a potential animal model to predict drug-related oculotoxicity at the preclinical stage. ${ }^{114}$ In this study, $3 \mathrm{dpf}$ zebrafish larvae were exposed to the known oculotoxic drugs (digoxin, gentamicin, ibuprofen, minoxidil, and quinine) for $48 \mathrm{~h}$, and toxicity was assessed using visual behaviour. These drugs resulted in damage to visual function in zebrafish, thereby confirming the potential toxicity of these drugs to the human eye.

In addition, many studies have been carried out to validate zebrafish assays for drug-toxicity assessment. In one of those, 27 drugs, 19 of which with known and 8 with no record of oculotoxicity in humans, were assessed in zebrafish using OMR. ${ }^{63}$ Of the 19 compounds, 13 drug compounds, including chlorpromazine, quinine, digoxin, AZ compound 1, deferoxamine, flecainide, ganciclovir, ibuprofen, minoxidil, thioridazine, and vardenafil, were found to be oculotoxic in zebrafish. This finding is supported by another similar study that tested nine known drug compounds with oculotoxicity and found that 7 out of the 9 compounds had adverse effect on visual function of zebrafish as well. ${ }^{117}$ Furthermore, zebrafish have been used extensively as a screening tool for small molecules that modulate vertebrate development. ${ }^{118}$ For example, small-molecule screens have identified compounds that can affect vascular development in the zebrafish retina. In one study, around 2000 small molecules were tested in zebrafish embryos followed by monitoring changes in retinal vasculature, and out of those, 5 compounds were identified, ${ }^{119}$ which suggests that these drugs may also contribute to the development of pathological changes to the retinal vasculature in human patients. These findings suggest that zebrafish models are successful in mirroring known oculotoxic characteristics of drugs in humans and have the potential to predict oculotoxicity profiles of novel drugs.

\section{Summary}

Visual impairment is a major health problem worldwide, and our understanding of the pathological mechanisms associated with disease is crucial for the development of novel therapeutic approaches. Likewise, the availability of animal models that closely mirror eye pathology but at the same time allow medium-throughput drug screening is desirable. In this context, zebrafish with their shortgeneration time, large clutch, ease of mutagenesis and genetic analysis, rapid eye development, and comparable eye morphology to humans seem ideally suited to address these needs. Similar phenotypes related to many human vision disorders have been reproduced in zebrafish, and for many available strains, their visual capacity has already been characterized using assays that exploit natural fish behaviour. Thus, the zebrafish model has the potential to open the door for a deeper understanding of ophthalmological disorders, as well as for the development of novel therapeutic agents.

\section{Conflict of interest}

The authors declare no conflict of interest.

\section{References}

1 Pascolini D, Mariotti SP. Global estimates of visual impairment: 2010. Br J Ophthalmol 2012; 96(5): 614-618.

2 Gordois A, Cutler H, Pezzullo L, Gordon K, Cruess A, Winyard $\mathrm{S}$ et al. An estimation of the worldwide economic and health burden of visual impairment. Glob Public Health 2012; 7(5): 465-481.

3 Brockerhoff SE, Hurley JB, Janssen-Bienhold U, Neuhauss SC, Driever W, Dowling JE. A behavioral screen for isolating zebrafish mutants with visual system defects. Proc Natl Acad Sci USA 1995; 92(23): 10545-10549.

4 Neuhauss SC, Biehlmaier O, Seeliger MW, Das T, Kohler K, Harris WA et al. Genetic disorders of vision revealed by a behavioral screen of 400 essential loci in zebrafish. J Neurosci 1999; 19(19): 8603-8615.

5 Neuhauss SC. Behavioral genetic approaches to visual system development and function in zebrafish. J Neurobiol 2003; 54(1): 148-160.

6 Muto A, Orger MB, Wehman AM, Smear MC, Kay JN, Page-McCaw PS et al. Forward genetic analysis of visual behavior in zebrafish. PLoS Genet 2005; 1(5): e66.

7 Bibliowicz J, Tittle RK, Gross JM. Toward a better understanding of human eye disease insights from the zebrafish, Danio rerio. Prog Mol Biol Transl Sci 2011; 100 287-330.

8 Mathias JR, Saxena MT, Mumm JS. Advances in zebrafish chemical screening technologies. Future Med Chem 2012; 4(14): 1811-1822.

9 Chakraborty C, Hsu CH, Wen ZH, Lin CS, Agoramoorthy G. Zebrafish: a complete animal model for in vivo drug discovery and development. Curr Drug Metab 2009; 10(2): 116-124.

10 Kari G, Rodeck U, Dicker AP. Zebrafish: an emerging model system for human disease and drug discovery. Clin Pharmacol Ther 2007; 82(1): 70-80.

11 Lin YY. Muscle diseases in the zebrafish. Neuromuscul Disord 2012; 22(8): 673-684.

12 Norton WH. Toward developmental models of psychiatric disorders in zebrafish. Front Neural Circuits 2013; 7: 79.

13 Ramakrishnan L. Looking within the zebrafish to understand the tuberculous granuloma. Adv Exp Med Biol 2013; 783: 251-266.

14 Amatruda JF, Shepard JL, Stern HM, Zon LI. Zebrafish as a cancer model system. Cancer Cell 2002; 1(3): 229-231. 
15 Lien CL, Harrison MR, Tuan TL, Starnes VA. Heart repair and regeneration: recent insights from zebrafish studies. Wound Repair Regen 2012; 20(5): 638-646.

16 Lohi O, Parikka M, Ramet M. The zebrafish as a model for paediatric diseases. Acta Paediatr 2013; 102(2): 104-110.

17 Bilotta J, Saszik S. The zebrafish as a model visual system. Int J Dev Neurosci 2001; 19(7): 621-629.

18 Villegas GM. Comparative ultrastructure of the retina in fish, monkey and man. Neurophysiologie und Psychophysik des Visuellen Systems/The Visual System: Neurophysiology and Psychophysics. Springer: Berlin, Germany; Heidelberg, Germany, 1961, pp 3-13.

19 Dowling J. The Retina: An Approachable Part of the Brain, Vol 18. Belknap Press of Harvard University Press: Cambridge, MA, USA, 1987, pp 12-32.

20 Curcio CA, Allen KA. Topography of ganglion cells in human retina. J Comp Neurol 1990; 300(1): 5-25.

21 Robinson J, Schmitt EA, Harosi FI, Reece RJ, Dowling JE. Zebrafish ultraviolet visual pigment: absorption spectrum, sequence, and localization. Proc Natl Acad Sci USA 1993; 90(13): 6009-6012.

22 Goldsmith P, Harris WA. The zebrafish as a tool for understanding the biology of visual disorders. Semin Cell Dev Biol 2003; 14(1): 11-18.

23 Fleisch VC, Neuhauss SC. Visual behavior in zebrafish. Zebrafish 2006; 3(2): 191-201.

24 Maurer CM, Huang YY, Neuhauss SC. Application of zebrafish oculomotor behavior to model human disorders. Rev Neurosci 2011; 22(1): 5-16.

25 Yousef YA, Finger PT. Optical coherence tomography of radiation optic neuropathy. Ophthalmic Surg Lasers Imaging 2012; 43(1): 6-12.

26 Schweitzer J, Gimnopoulos D, Lieberoth BC, Pogoda HM, Feldner J, Ebert A et al. Contactin1a expression is associated with oligodendrocyte differentiation and axonal regeneration in the central nervous system of zebrafish. Mol Cell Neurosci 2007; 35(2): 194-207.

27 Zou S, Tian C, Ge S, Hu B. Neurogenesis of retinal ganglion cells is not essential to visual functional recovery after optic nerve injury in adult zebrafish. PLoS One 2013; 8(2): e57280.

28 Lagnado L. Retinal processing: amacrine cells keep it short and sweet. Curr Biol 1998; 8(17): R598-R600.

29 Raymond PA, Barthel LK, Rounsifer ME, Sullivan SA, Knight JK. Expression of rod and cone visual pigments in goldfish and zebrafish: a rhodopsin-like gene is expressed in cones. Neuron 1993; 10(6): 1161-1174.

30 Branchek T, Bremiller R. The development of photoreceptors in the zebrafish, Brachydanio rerio. I. Structure. J Comp Neurol 1984; 224(1): 107-115.

31 Saszik S, Bilotta J, Givin CM. ERG assessment of zebrafish retinal development. Vis Neurosci 1999; 16(5): 881-888.

32 Moyano M, Porteros A, Dowling JE. The effects of nicotine on cone and rod b-wave responses in larval zebrafish. Vis Neurosci 2013; 30(4): 141-145.

33 Baylor DA. Photoreceptor signals and vision. Proctor lecture. Invest Ophthalmol Vis Sci 1987; 28(1): 34-49.

34 Schmitt EA, Dowling JE. Early eye morphogenesis in the zebrafish, Brachydanio rerio. J Comp Neurol 1994; 344(4): 532-542.

35 Schmitt EA, Dowling JE. Early retinal development in the zebrafish, Danio rerio: light and electron microscopic analyses. J Comp Neurol 1999; 404(4): 515-536.
36 Malicki J. Development of the retina. Methods Cell Biol 1999; 59: 273-299.

$37 \mathrm{Hu}$ M, Easter SS. Retinal neurogenesis: the formation of the initial central patch of postmitotic cells. Dev Biol 1999; 207(2): 309-321.

38 Fadool JM, Dowling JE. Zebrafish: a model system for the study of eye genetics. Prog Retin Eye Res 2008; 27(1): 89-110.

39 Tsujikawa M, Malicki J. Genetics of photoreceptor development and function in zebrafish. Int J Dev Biol 2004; 48(8-9): 925-934.

40 Easter Jr SS, Nicola GN. The development of vision in the zebrafish (Danio rerio). Dev Biol 1996; 180(2): 646-663.

41 Easter Jr SS, Nicola GN. The development of eye movements in the zebrafish (Danio rerio). Dev Psychobiol 1997; 31(4): 267-276.

42 Kitambi SS, Chandrasekar G, Addanki VK. Teleost fish-a powerful models for studying development, function and diseases of the human eye. Curr Sci 2011; 100(12): 1815.

43 Renninger SL, Schonthaler HB, Neuhauss SC, Dahm R. Investigating the genetics of visual processing, function and behaviour in zebrafish. Neurogenetics 2011; 12(2): 97-116.

44 Brockerhoff SE. Measuring the optokinetic response of zebrafish larvae. Nat Protoc 2006; 1(5): 2448-2451.

45 Huang YY, Neuhauss SC. The optokinetic response in zebrafish and its applications. Front Biosci 2008; 13 1899-1916.

46 Huber-Reggi SP, Mueller KP, Neuhauss SC. Analysis of optokinetic response in zebrafish by computer-based eye tracking. Methods Mol Biol 2013; 935: 139-160.

47 Mueller KP, Neuhauss SC. Quantitative measurements of the optokinetic response in adult fish. J Neurosci Methods 2010; 186(1): 29-34.

48 Link BA, Gray MP, Smith RS, John SW. Intraocular pressure in zebrafish: comparison of inbred strains and identification of a reduced melanin mutant with raised IOP. Invest Ophthalmol Vis Sci 2004; 45(12): 4415-4422.

49 Brockerhoff SE, Hurley JB, Niemi GA, Dowling JE. A new form of inherited red-blindness identified in zebrafish. J Neurosci 1997; 17(11): 4236-4242.

50 Bilotta J. Effects of abnormal lighting on the development of zebrafish visual behavior. Behav Brain Res 2000; 116(1): 81-87.

51 Quentin B, Moore RH. Behavior and Cognition. Biology of Fishes, 3rd edn. Taylor \& Francis Group: New York, USA; Abingdon, UK, 2007, pp 409-433.

52 Bilotta J, Saszik S, Givin CM, Hardesty HR, Sutherland SE. Effects of embryonic exposure to ethanol on zebrafish wvisual function. Neurotoxicol Teratol 2002; 24(6): 759-766

53 Kimmel CB, Patterson J, Kimmel RO. The development and behavioral characteristics of the startle response in the zebra fish. Dev Psychobiol 1974; 7(1): 47-60.

54 Weber DN, Connaughton VP, Dellinger JA, Klemer D, Udvadia A, Carvan 3rd MJ. Selenomethionine reduces visual deficits due to developmental methylmercury exposures. Physiol Behav 2008; 93(1-2): 250-260.

55 Emran F, Rihel J, Adolph AR, Wong KY, Kraves S, Dowling JE. OFF ganglion cells cannot drive the optokinetic reflex in zebrafish. Proc Natl Acad Sci USA 2007; 104(48): 19126-19131.

56 Emran F, Rihel J, Dowling JE. A behavioral assay to measure responsiveness of zebrafish to changes in light intensities. J Vis Exp 2008; (20). pii. 923; doi:10.3791/923. 
$57 \mathrm{Li} \mathrm{L}$, Dowling JE. A dominant form of inherited retinal degeneration caused by a non-photoreceptor cell-specific mutation. Proc Natl Acad Sci USA 1997; 94(21): 11645-11650.

58 Bianco IH, Kampff AR, Engert F. Prey capture behavior evoked by simple visual stimuli in larval zebrafish. Front Syst Neurosci 2011; 5: 101.

59 Li L. Zebrafish mutants: behavioral genetic studies of visual system defects. Dev Dyn 2001; 221(4): 365-372.

$60 \mathrm{Li} \mathrm{L}$, Dowling JE. Zebrafish visual sensitivity is regulated by a circadian clock. Vis Neurosci 1998; 15(5): 851-857.

$61 \mathrm{Li} \mathrm{L}$, Dowling JE. Disruption of the olfactoretinal centrifugal pathway may relate to the visual system defect in night blindness b mutant zebrafish. J Neurosci 2000; 20(5): 1883-1892.

62 Luca RM, Gerlai R. Animated bird silhouette above the tank: acute alcohol diminishes fear responses in zebrafish Behav Brain Res 2012; 229(1): 194-201.

63 Richards FM, Alderton WK, Kimber GM, Liu Z, Strang I, Redfern WS et al. Validation of the use of zebrafish larvae in visual safety assessment. J Pharmacol Toxicol Methods 2008; 58(1): 50-58.

64 Lang GK. Ophthalmology A Pocket Textbook Atlas. 2nd ed. Thieme: New York, NY, USA, 2007.

65 Li XQ, Cai HC, Zhou SY, Yang JH, Xi YB, Gao XB et al. A novel mutation impairing the tertiary structure and stability of gammaC-crystallin (CRYGC) leads to cataract formation in humans and zebrafish lens. Hum Mutat 2012; 33(2): 391-401.

66 Reis LM, Tyler RC, Muheisen S, Raggio V, Salviati L, Han DP et al. Whole exome sequencing in dominant cataract identifies a new causative factor, CRYBA2, and a variety of novel alleles in known genes. Hum Genet 2013; 132(7): 761-770.

67 Andley UP. Crystallins in the eye: function and pathology. Prog Retin Eye Res 2007; 26(1): 78-98.

68 Jonasova K, Kozmik Z. Eye evolution: lens and cornea as an upgrade of animal visual system. Semin Cell Dev Biol 2008; 19(2): 71-81.

69 Graw J. The crystallins: genes, proteins and diseases. Biol Chem 1997; 378(11): 1331-1348.

70 Graw J. Genetics of crystallins: cataract and beyond. Exp Eye Res 2009; 88(2): 173-189.

71 Goishi K, Shimizu A, Najarro G, Watanabe S, Rogers R, Zon LI et al. AlphaA-crystallin expression prevents gamma-crystallin insolubility and cataract formation in the zebrafish cloche mutant lens. Development 2006; 133(13): 2585-2593.

72 Semina EV, Ferrell RE, Mintz-Hittner HA, Bitoun P, Alward WL, Reiter RS et al. A novel homeobox gene PITX3 is mutated in families with autosomal-dominant cataracts and ASMD. Nat Genet 1998; 19(2): 167-170.

73 Semina EV, Brownell I, Mintz-Hittner HA, Murray JC, Jamrich M. Mutations in the human forkhead transcription factor FOXE3 associated with anterior segment ocular dysgenesis and cataracts. Hum Mol Genet 2001; 10(3): 231-236.

74 Dutta S, Dietrich JE, Aspock G, Burdine RD, Schier A, Westerfield $\mathrm{M}$ et al. pitx3 defines an equivalence domain for lens and anterior pituitary placode. Development 2005; 132(7): 1579-1590.

75 Swindell EC, Zilinski CA, Hashimoto R, Shah R, Lane ME, Jamrich M. Regulation and function of foxe3 during early zebrafish development. Genesis 2008; 46(3): 177-183.
76 Chen CC, Yeh LK, Liu CY, Kao WW, Samples JR, Lin SJ et al. Morphological differences between the trabecular meshworks of zebrafish and mammals. Curr Eye Res 2008; 33(1): 59-72.

77 Gray MP, Smith RS, Soules KA, John SW, Link BA. The aqueous humor outflow pathway of zebrafish. Invest Ophthalmol Vis Sci 2009; 50(4): 1515-1521.

78 Wong TY, Klein BE, Klein R, Knudtson M, Lee KE. Refractive errors, intraocular pressure, and glaucoma in a white population. Ophthalmology 2003; 110(1): 211-217.

79 Tamimi Y, Skarie JM, Footz T, Berry FB, Link BA, Walter MA. FGF19 is a target for FOXC1 regulation in ciliary body-derived cells. Hum Mol Genet 2006; 15(21): 3229-3240.

80 Berry FB, Skarie JM, Mirzayans F, Fortin Y, Hudson TJ, Raymond $\mathrm{V}$ et al. FOXC1 is required for cell viability and resistance to oxidative stress in the eye through the transcriptional regulation of FOXO1A. Hum Mol Genet 2008; 17(4): 490-505.

81 Skarie JM, Link BA. FoxC1 is essential for vascular basement membrane integrity and hyaloid vessel morphogenesis. Invest Ophthalmol Vis Sci 2009; 50(11): 5026-5034.

82 McMahon C, Semina EV, Link BA. Using zebrafish to study the complex genetics of glaucoma. Comp Biochem Physiol C Toxicol Pharmacol 2004; 138(3): 343-350.

83 Stujenske JM, Dowling JE, Emran F. The bugeye mutant zebrafish exhibits visual deficits that arise with the onset of an enlarged eye phenotype. Invest Ophthalmol Vis Sci 2011; 52(7): 4200-4207.

84 Veth KN, Willer JR, Collery RF, Gray MP, Willer GB, Wagner DS et al. Mutations in zebrafish lrp2 result in adult-onset ocular pathogenesis that models myopia and other risk factors for glaucoma. PLoS Genet 2011; 7(2): e1001310.

85 Lim LS, Mitchell P, Seddon JM, Holz FG, Wong TY. Age-related macular degeneration. Lancet 2012; 379(9827): $1728-1738$.

86 Jager RD, Mieler WF, Miller JW. Age-related macular degeneration. N Engl J Med 2008; 358(24): 2606-2617.

87 Jensen LD, Rouhi P, Cao Z, Lanne T, Wahlberg E, Cao Y. Zebrafish models to study hypoxia-induced pathological angiogenesis in malignant and nonmalignant diseases. Birth Defects Res C Embryo Today 2011; 93(2): 182-193.

88 Cao Z, Jensen LD, Rouhi P, Hosaka K, Lanne T, Steffensen JF et al. Hypoxia-induced retinopathy model in adult zebrafish. Nat Protoc 2010; 5(12): 1903-1910.

89 Cao R, Jensen LD, Soll I, Hauptmann G, Cao Y. Hypoxiainduced retinal angiogenesis in zebrafish as a model to study retinopathy. PLoS One 2008; 3(7): e2748.

90 Biehlmaier O, Neuhauss SC, Kohler K. Double cone dystrophy and RPE degeneration in the retina of the zebrafish gnn mutant. Invest Ophthalmol Vis Sci 2003; 44(3): 1287-1298.

91 Whiting DR, Guariguata L, Weil C, Shaw J. IDF diabetes atlas: global estimates of the prevalence of diabetes for 2011 and 2030. Diabetes Res Clin Pract 2011; 94(3): 311-321.

92 Wild S, Roglic G, Green A, Sicree R, King H. Global prevalence of diabetes: estimates for the year 2000 and projections for 2030. Diabetes Care 2004; 27(5): 1047-1053.

93 Stitt AW, Lois N, Medina RJ, Adamson P, Curtis TM. Advances in our understanding of diabetic retinopathy. Clin Sci (Lond) 2013; 125(1): 1-17. 
94 Bandello F, Lattanzio R, Zucchiatti I, Del Turco C. Pathophysiology and treatment of diabetic retinopathy. Acta Diabetol 2013; 50(1): 1-20.

95 Alvarez Y, Cederlund ML, Cottell DC, Bill BR, Ekker SC, Torres-Vazquez J et al. Genetic determinants of hyaloid and retinal vasculature in zebrafish. BMC Dev Biol 2007; 7: 114

96 Gleeson M, Connaughton V, Arneson LS. Induction of hyperglycaemia in zebrafish (Danio rerio) leads to morphological changes in the retina. Acta Diabetol 2007; 44(3): 157-163.

97 Alvarez Y, Chen K, Reynolds AL, Waghorne N, O'Connor JJ Kennedy BN. Predominant cone photoreceptor dysfunction in a hyperglycaemic model of non-proliferative diabetic retinopathy. Dis Model Mech 2010; 3(3-4): 236-245.

98 Thomas MG, Crosier M, Lindsay S, Kumar A, Thomas S, Araki $\mathrm{M}$ et al. The clinical and molecular genetic features of idiopathic infantile periodic alternating nystagmus. Brain 2011; 134(Pt 3): 892-902.

99 Self J, Lotery A. A review of the molecular genetics of congenital idiopathic nystagmus (CIN). Ophthalmic Genet 2007; 28(4): 187-191.

100 Huber-Reggi SP, Chen CC, Grimm L, Straumann D, Neuhauss SC, Huang MY. Severity of infantile nystagmus syndrome-like ocular motor phenotype is linked to the extent of the underlying optic nerve projection defect in zebrafish belladonna mutant. J Neurosci 2012; 32(50): 18079-18086.

101 Rachel RA, Li T, Swaroop A. Photoreceptor sensory cilia and ciliopathies: focus on CEP290, RPGR and their interacting proteins. Cilia 2012; 1(1): 22.

102 Vincensini L, Blisnick T, Bastin P. 1001 model organisms to study cilia and flagella. Biol Cell 2011; 103(3): 109-130.

103 Pazour GJ, Baker SA, Deane JA, Cole DG, Dickert BL, Rosenbaum JL et al. The intraflagellar transport protein, IFT88, is essential for vertebrate photoreceptor assembly and maintenance. J Cell Biol 2002; 157(1): 103-113.

104 Insinna C, Besharse JC. Intraflagellar transport and the sensory outer segment of vertebrate photoreceptors. Dev Dyn 2008; 237(8): 1982-1992.

105 Bahadori R, Huber M, Rinner O, Seeliger MW, Geiger-Rudolph S, Geisler R et al. Retinal function and morphology in two zebrafish models of oculo-renal syndromes. Eur J Neurosci 2003; 18(6): 1377-1386.

106 Jurisch-Yaksi N, Rose AJ, Lu H, Raemaekers T, Munck S, Baatsen $\mathrm{P}$ et al. Rer1p maintains ciliary length and signaling by regulating gamma-secretase activity and Foxj1a levels. J Cell Biol 2013; 200(6): 709-720.
107 Gestri G, Link BA, Neuhauss SC. The visual system of zebrafish and its use to model human ocular diseases. Dev Neurobiol 2012; 72(3): 302-327.

108 Starr CJ, Kappler JA, Chan DK, Kollmar R, Hudspeth AJ. Mutation of the zebrafish choroideremia gene encoding Rab escort protein 1 devastates hair cells. Proc Natl Acad Sci USA 2004; 101(8): 2572-2577.

109 Jacobson SG, Cideciyan AV, Sumaroka A, Aleman TS, Schwartz SB, Windsor EA et al. Remodeling of the human retina in choroideremia: rab escort protein 1 (REP-1) mutations. Invest Ophthalmol Vis Sci 2006; 47(9): 4113-4120.

110 Moosajee M, Tulloch M, Baron RA, Gregory-Evans CY, Pereira-Leal JB, Seabra MC. Single choroideremia gene in nonmammalian vertebrates explains early embryonic lethality of the zebrafish model of choroideremia. Invest Ophthalmol Vis Sci 2009; 50(6): 3009-3016.

111 Bahadori R, Rinner O, Schonthaler HB, Biehlmaier O, Makhankov YV, Rao P et al. The Zebrafish fade out mutant: a novel genetic model for Hermansky-Pudlak syndrome. Invest Ophthalmol Vis Sci 2006; 47(10): 4523-4531.

112 Santoriello C, Zon LI. Hooked! Modeling human disease in zebrafish. J Clin Invest 2012; 122(7): 2337-2343.

113 Santaella RM, Fraunfelder FW. Ocular adverse effects associated with systemic medications: recognition and management. Drugs 2007; 67(1): 75-93.

114 Deeti S, O'Farrell S, Kennedy BN. Early safety assessment of human oculotoxic drugs using the zebrafish visualmotor response. J Pharmacol Toxicol Methods 2013; 69(1): 1-8.

115 Rubinstein AL. Zebrafish assays for drug toxicity screening. Expert Opin Drug Metab Toxicol 2006; 2(2): 231-240.

116 Eimon PM, Rubinstein AL. The use of in vivo zebrafish assays in drug toxicity screening. Expert Opin Drug Metab Toxicol 2009; 5(4): 393-401.

117 Berghmans S, Butler P, Goldsmith P, Waldron G, Gardner I, Golder $\mathrm{Z}$ et al. Zebrafish based assays for the assessment of cardiac, visual and gut function-potential safety screens for early drug discovery. J Pharmacol Toxicol Methods 2008; 58(1): 59-68.

118 Peterson RT, Link BA, Dowling JE, Schreiber SL. Small molecule developmental screens reveal the logic and timing of vertebrate development. Proc Natl Acad Sci USA 2000; 97(24): 12965-12969.

119 Kitambi SS, McCulloch KJ, Peterson RT, Malicki JJ. Small molecule screen for compounds that affect vascular development in the zebrafish retina. Mech Dev 2009; 126(5-6): 464-477. 